\title{
F11 Gene Duplication Causes Elevated FXI Plasma Levels and Is a Risk for Venous Thrombosis
}

\author{
Christine Van Laer ${ }^{1,2}$ Kathelijne Peerlinck ${ }^{1,3}$ Marc Jacquemin ${ }^{1,2}$ Chantal Thys ${ }^{1}$ Kate Downes ${ }^{4,5}$ \\ Veerle Labarque $^{1,6}$ Kathleen Freson ${ }^{1}$
}

${ }^{1}$ Department of Cardiovascular Sciences, Center for Molecular and Vascular Biology, University of Leuven, Leuven, Belgium

${ }^{2}$ Clinical Department of Laboratory Medicine, University Hospitals Leuven, Leuven, Belgium

${ }^{3}$ Vascular Medicine and Hemostasis, University Hospitals Leuven, Leuven, Belgium

${ }^{4}$ East Genomic Laboratory Hub, Cambridge University Hospitals NHS Foundation Trust, Cambridge, United Kingdom

${ }^{5}$ Department of Haematology, Cambridge Biomedical Campus, University of Cambridge, Cambridge, United Kingdom

${ }^{6}$ Department of Pediatrics, Pediatric Hemato-Oncology, University Hospitals Leuven, Leuven, Belgium

Thromb Haemost 2022;122:1058-1060.

Coagulation factor XI (FXI) is the zymogen of a plasma protease encoded by $F 11$ located on 4q35.2 and is mainly synthesized by hepatocytes. ${ }^{1,2}$ FXI is activated by coagulation factor XIIa and plays an important role in the intrinsic coagulation pathway of thrombin generation resulting in fibrin clot formation. ${ }^{3,4}$ Bleeding problems can be due to FXI deficiency, but without clear correlations between FXI plasma levels and bleeding severity. ${ }^{2}$ FXI deficiency is a rare disorder ( 1 in 1 million in white population) but with a higher prevalence in some ethnic groups, for instance $9 \%$ in Ashkenazi Jews. ${ }^{5}$ In contrast, elevated plasma FXI levels are correlated with a higher risk for venous thromboembolism (VTE) and ischemic stroke. ${ }^{6-9}$ There is no clear correlation between FXI levels and their contribution to myocardial infarction. ${ }^{8}$ Different reports describe an association between genetic variability in F11 locus and venous thrombosis with elevated FXI levels. ${ }^{10,11}$ These genetic factors are noncoding frequent single nucleotide polymorphisms (SNPs) located in F11 introns, e.g., rs2036914 and rs2289252. ${ }^{10,12,13}$ Due to these insights related to a potential prothrombotic role of FXI, there is a growing interest in targeting FXI with new anticoagulant therapies. ${ }^{8}$

We here describe a family with inherited thrombophilia ( - Fig. 1A). The 58-year-old proband (II.6) with a history of recurrent VTE and negative routine thrombophilia laboratory screening was screened in the ThromboGenomics study using a targeted panel for known genes associated with
Address for correspondence Kathleen Freson, PhD, Center for Molecular and Vascular Biology, Herestraat 49 B911, 3000 Leuven, Belgium (e-mail: kathleen.freson@kuleuven.be).

bleeding and thrombosis. ${ }^{14}$ A F11 duplication was found while no other pathogenic variants could be detected in known thrombophilia genes (-Fig. 1B). As only F11 exonic regions were captured, the exact size of the duplication remains unknown but it involves at least the complete F11 gene (arr[GRCh37] 4q35.2:(187185980-187209984)x3). Cosegregation analysis of this duplication was performed in the family using real-time polymerase chain reaction to calculate the gene dosage ratio for F11 and its neighboring genes FGG and KLKB1 (upstream) and FAT1 (downstream) (-Fig. 1C, -Supplementary Table S1, available in the online version). In addition, the $F 11$ gene duplication was confirmed in the proband (II.6) and his brother (II.5) using Multiplex Ligation-dependent Probe Amplification (MLPA). For this, $300 \mathrm{ng}$ of DNA from II.5 and II.6 and four normal control samples were assayed using the MRC-Holland MLPA kit for the F11 gene (SALSA MLPA Probemix P440-A2 F10 + F11) as per manufacturer's instructions. Fragments were analyzed using an ABI 3730XL sequencer and interpreted using GeneMarker software (SoftGenetics).

FXI plasma activity levels were measured with the ACL TOP analyzer (Werfen, Brussels, Belgium) according to the manufacturer's recommendations. For patients receiving direct oral anticoagulant (DOAC) therapy, sample pretreatment with DOAC-STOP (Nodia, Boom, Belgium) was performed. The normal range for FXI was 70 to $130 \%$. Control experiments showed received

May 31, 2021

accepted

October 5, 2021

published online

November 15, 2021 (c) 2021. Thieme. All rights reserved. Georg Thieme Verlag KG,

Rüdigerstraße 14,

70469 Stuttgart, Germany
DOI https://doi.org/

10.1055/s-0041-1739363. ISSN 0340-6245. 


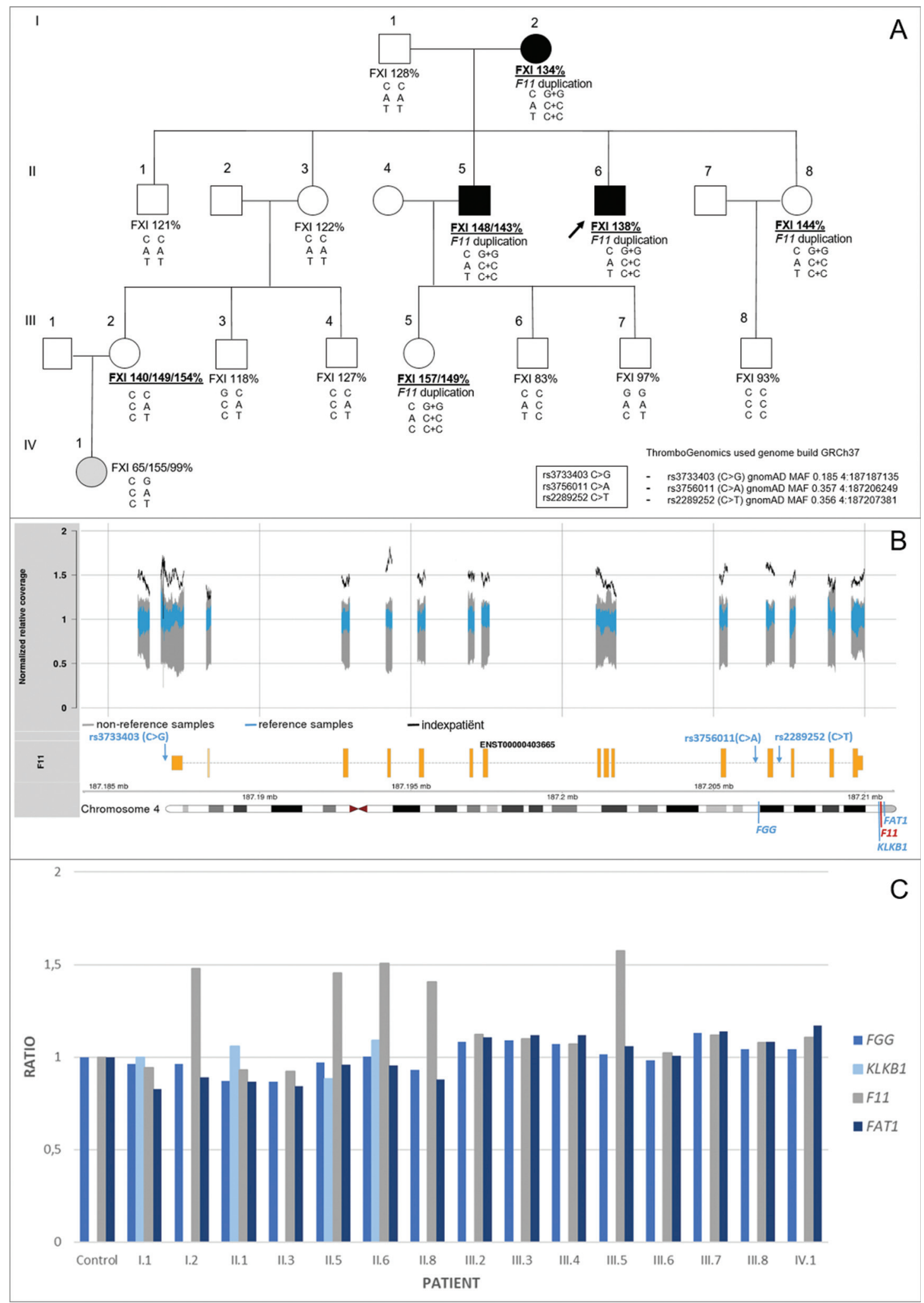

Fig. 1 (A) Pedigree of the VTE patients (black symbols and index indicated with arrow), case IV.1 with a neonatal arteria cerebri media infarct (gray symbols), and their unaffected relatives (white symbols). Five family members carry the $F 11$ duplication as indicated. FXI plasma values are indicated (normal values between 70 and 130\%). The analysis results for Sanger sequencing of F11 SNP rs 3733403 (C>G), rs 3756011 (C > A), and rs2289252 (C>T) are indicated. (B) Coverage profile for the F11 gene obtained for reference samples and the index patient II.6 (black line) showing significantly higher coverage over the complete $F 11$ locus. The locations of studied SNP rs3733403, rs3756011, and rs2289252 are indicated on the map for F11. The locations of studied genes FGG (exon 1), KLKB1 (intron 6-7), F11 (intron 10-11), and FAT1 (exon 10) are indicated on the chromosome 4 map. (C) Gene dosage ratios for F11, FGG, KLKB1, and FAT1 (on Y-axis) of different patients (on $X$-axis) determined with real-time PCR. A ratio of 1.5 is indicative for a gene duplication. PCR, polymerase chain reaction; SNP, single nucleotide polymorphism; VTE, venous thromboembolism. 
that pretreatment of the samples with DOAC-STOP did not bias the FXI assay (data not shown). All five family members (I.2, II.5, II.6, II.8, III.5) carrying the F11 duplication have elevated FXI plasma levels (-Fig. 1A). The proband (II.6) and his brother (II.5) both had a history of unexplained VTE while his sister (II.8) had no history of thrombosis ( - Supplementary Table S2, available in the online version). Their mother (I.2) had pulmonary embolism at the age of 70. Patient III.5, a 30-year-old woman, had no history of thrombosis. Remarkably, elevated FXI levels were also measured for III.2 and IV.1, who have no F11 duplication (- Fig. 1C). The 33-year-old woman III.2 is healthy while her daughter (IV.1) presented with a neonatal arteria cerebri media infarct with subarachnoid and plexus choroideus hemorrhage. Her FXI level was normal for age at birth (65\%), while it was temporary elevated (155\%) at the age of 3 years but normalized on a control sample (99\%). She carried no pathogenic variants in any of the known thrombosis and hemostasis genes using a panel test. ${ }^{15}$ Sanger sequencing of F11 SNP rs3733403, rs3756011, and rs2289252 in members of this pedigree showed that II.3 and III.2 inherited the other F11 allele from affected (grand)mother I.2 than the allele present in index case II.6 and the other family members with duplicated F11 (-Fig. 1A, - Supplementary Table S3, and -Supplementary Figs. S1-S3, available in the online version). Family members III.7 and III.8 with normal FXI levels and no F11 deletion carried other F11 SNP than their affected father and mother, respectively, pointing out the presence of frequent chromosomal rearrangements on the duplicated $F 11$ allele. The genetic variant rs2289252 was previously associated with elevated FXI levels and contributed to the risk of VTE, but these values were never higher than the upper value of the normal range. ${ }^{10,16,17}$

This report is the first described case of a family with F11 gene duplication leading to elevated FXI activity levels. As described above, these increased FXI activity levels can be an additional risk factor for thrombosis. In this family the presence of $F 11$ gene duplication is important for thrombophilia risk management.

\section{Funding}

This work was supported by KU Leuven BOF grant C14/19/ 096, FWO grant G072921N, and research grants from Novo Nordisk, CSL Behring, Bayer, and Swedish Orphan Biovitrum AB (Sobi).

\section{Conflict of Interest}

None declared.

\section{Acknowledgments}

We thank Tom Legerton at the Cambridge University Hospitals Genomic Laboratory for technical assistance.

\section{References}

1 Mohammed BM, Matafonov A, Ivanov I, et al. An update on factor XI structure and function. Thromb Res 2018;161:94-105

2 Visser M, Heitmeier S, Ten Cate H, Spronk HMH. Role of factor XIa and plasma kallikrein in arterial and venous thrombosis. Thromb Haemost 2020;120(06):883-993

3 Puy C, Rigg RA, McCarty OJ. The hemostatic role of factor XI. Thromb Res 2016;141(Suppl 2):S8-S11

4 Matafonov A, Sarilla S, Sun MF, et al. Activation of factor XI by products of prothrombin activation. Blood 2011;118(02): 437-445

5 Esteban J, de la Morena-Barrio ME, Salloum-Asfar S, et al. High incidence of FXI deficiency in a Spanish town caused by 11 different mutations and the first duplication of $\mathrm{F} 11$ : results from the Yecla study. Haemophilia 2017;23(06):e488-e496

6 Meijers JC, Tekelenburg WL, Bouma BN, Bertina RM, Rosendaal FR. High levels of coagulation factor XI as a risk factor for venous thrombosis. N Engl J Med 2000;342(10):696-701

7 Kyrle PA, Eischer L, Šinkovec H, Eichinger S. Factor XI and recurrent venous thrombosis: an observational cohort study. J Thromb Haemost 2019;17(05):782-786

8 Fredenburgh JC, Gross PL, Weitz JI. Emerging anticoagulant strategies. Blood 2017;129(02):147-154

9 Gill D, Georgakis MK, Laffan M, et al. Genetically determined FXI (factor XI) levels and risk of stroke. Stroke 2018;49(11): 2761-2763

10 Li Y, Bezemer ID, Rowland CM, et al. Genetic variants associated with deep vein thrombosis: the F11 locus. J Thromb Haemost 2009;7(11):1802-1808

11 Martin-Fernandez L, Gavidia-Bovadilla G, Corrales I, et al. Next generation sequencing to dissect the genetic architecture of KNG1 and F11 loci using factor XI levels as an intermediate phenotype of thrombosis. PLoS One 2017;12(04):e0176301

12 Austin H, De Staercke C, Lally C, Bezemer ID, Rosendaal FR, Hooper WC. New gene variants associated with venous thrombosis: a replication study in White and Black Americans. J Thromb Haemost 2011;9(03):489-495

13 de Haan HG, van Hylckama Vlieg A, Lotta LA, et al; INVENT consortium. Targeted sequencing to identify novel genetic risk factors for deep vein thrombosis: a study of 734 genes. J Thromb Haemost 2018;16(12):2432-2441

14 Downes K, Megy K, Duarte D, et al; NIHR BioResource. Diagnostic high-throughput sequencing of 2396 patients with bleeding, thrombotic, and platelet disorders. Blood 2019;134(23): 2082-2091

15 Megy K, Downes K, Simeoni I, et al; Subcommittee on Genomics in Thrombosis and Hemostasis. Curated disease-causing genes for bleeding, thrombotic, and platelet disorders: communication from the SSC of the ISTH. J Thromb Haemost 2019;17(08): 1253-1260

16 Bruzelius M, Bottai M, Sabater-Lleal M, et al. Predicting venous thrombosis in women using a combination of genetic markers and clinical risk factors. J Thromb Haemost 2015;13(02): 219-227

17 Bruzelius M, Ljungqvist M, Bottai M, et al. F11 is associated with recurrent VTE in women. A prospective cohort study. Thromb Haemost 2016;115(02):406-414 